\section{Haploidentical hematopoietic stem cell transplantation in a myelofibrosis patient with primary graft failure}

\author{
Cristina Tecchio, ${ }^{1}$ Angelo Andreini, ${ }^{1}$ \\ Claudio Costantini, ${ }^{1}$ Alberto Zamò, ${ }^{2}$ \\ Donata de Sabata, ${ }^{1}$ Fiorenza Aprili, ${ }^{3}$ \\ Roberta Galavotti, ${ }^{4}$ \\ Emanuele Guardalben, ${ }^{1}$ \\ Fabio Benedetti ${ }^{1}$
}

${ }^{1}$ Section of Hematology and Bone Marrow Transplant Unit, Department of Medicine, ${ }^{2}$ Section of Pathology and Diagnostics, Department of Pathology, ${ }^{3}$ Clinical Biochemistry Laboratory, Department of Life and Reproduction Sciences, ${ }^{4}$ Section of Biology and Genetics, Department of Mother and Child, University of Verona, Italy

\begin{abstract}
The prognosis of patients affected by myelofibrosis (MF) is usually dismal and allogeneic hematopoietic stem cell transplantation (HSCT) remains the only cure. The number of HSCTs in MF patients has recently increased. However, a major obstacle is still represented by primary graft failure (PGF). Currently there are no definitive guidelines for the treatment of PGF and a second HSCT can be performed only when an allogeneic donor is rapidly available. Herein we report on a MF patient with PGF after an unrelated HSCT, who was rescued by a non-myeloablative, unmanipulated, haploidentical HSCT that resulted in persistent engraftment and bone-marrow fibrosis regression, but not in a long-term disease control. Based on this experience we briefly review the role of different conditioning regimens and hematopoietic stem cell sources in the setting of HSCT for MF patients with PGF. The role of haploidentical donors in MF patients lacking HLAmatched relatives is also discussed.
\end{abstract}

\section{Introduction}

Myelofibrosis (MF) is a pathological condition caused by the clonal proliferation of a pluripotent stem cell. It is characterized by a deregulated kinase signaling, and an abnormal release of cytokines, the latter being responsible for the induction of bonemarrow (BM) fibrosis. ${ }^{1} \mathrm{MF}$ is associated with three main driver mutations namely the $V 617 F$ mutation of the Janus Kinase 2
(JAK2) gene (in $\sim 60 \%$ of cases), and mutations of the calreticulin (CARL) and the myeloproliferative leukemia protein $(M P L)$ genes (in $\sim 30 \%$ and $8 \%$ of cases, respectively). ${ }^{1,2} \mathrm{MF}$ can arise de novo as primary myelofibrosis (PMF) or following essential thrombocythemia or polycythemia vera. Clinical presentation of MF includes blood cytopenia, leukoerithroblastosis, extramedullary hematopoiesis, progressive splenomegaly, and systemic symptoms. ${ }^{3}$ In spite of the recent developments of targeted therapies (e.g., JAK1/2 inhibitors), the prognosis of MF patients is usually dismal and the allogeneic hematopoietic stem cell transplantation (HSCT) remains the only cure. ${ }^{3}$ Improvements in patient selection, conditioning strategies and timing, have recently contributed to increase the number of HSCTs in MF patients. ${ }^{4}$ However, one of the major obstacles for a successful HSCT is represented by primary graft failure (PGF), which has been reported in $2 \%$ to $25 \%$ of MF patients. ${ }^{1}$

PGF is caused by the absence of an initial cell donor engraftment and is defined as the persistence of severe neutropenia together with red blood cells and platelets transfusion dependence after the HSCT conditioning. It is associated with a considerable morbidity and mortality, mainly driven by severe infections. A number of factors contribute to PGF, including hematopoietic stem cell (HSC) source, conditioning regimen, HLA disparity, AB0 mismatches, and infections. ${ }^{5}$ In myeloproliferative neoplasms possible causes of PGF may also include a defective BM stroma, splenic consumption of infused HSCs and allo-immunization following multiple transfusions. ${ }^{5}$ In MF patients increased levels of plasma cytokines (mostly TNF- $\alpha$ ) may also exert a cytotoxic activity against HSCs. ${ }^{1}$

Worthy of note BM fibrosis has not been clearly proven to correlate with PGF, but rather a consequence of an altered cytokine milieu able to reverse upon the replacement of the abnormal hematopoietic system. ${ }^{6}$ In the absence of definitive guidelines for the treatment of PGF patients may undergo a second HSCT, which can be performed using the same or another allogeneic donor when available.

\section{Case Report}

A 54-year-old woman was referred to our Institution on December 2012 with painful splenomegaly and systemic symptoms. Blood tests showed leukocytosis $\left(24 \times 10^{9} / \mathrm{L}\right)$ and thrombocytopenia $\left(97 \times 10^{9} / \mathrm{L}\right)$. The circulating CD34+ cells
Correspondence: Cristina Tecchio, Department of Medicine, Hematology and Bone Marrow Transplant Unit, University of Verona, Piazzale L.A. Scuro 10, 37134 Verona, Italy.

Tel.: +39.045.8124902 - Fax: +39.045.8124104.

E-mail: cristina.tecchio@univr.it

Key words: primary graft failure, haploidentical hematopoietic stem cell transplantation, myelofibrosis.

Contributions: FB, CT, AA, and EG planned the treatment schedule; AZ, DdS, FA and RG performed the research; CT and $\mathrm{CC}$ wrote the paper.

Conflict of interest: the authors declare no potential conflict of interest.

Received for publication: 13 February 2017.

Revision received: 6 October 2017.

Accepted for publication: 13 October 2017.

This work is licensed under a Creative Commons Attribution-NonCommercial 4.0 International License (CC BY-NC 4.0).

(C) Copyright C. Tecchio et al., 2017

Licensee PAGEPress, Italy

Hematology Reports 2017; 9:7091

doi:10.4081/hr:2017.7091

were $2.4 \%$. The BM aspirate was not evaluable due to the poor cellularity, while the BM biopsy was consistent with PMF. The karyotype was normal and the molecular analysis revealed the presence of the JAK2 $V 617 F$ mutation. The patient was diagnosed with an intermediate-1 PMF according to the International Prognostic Scoring System. ${ }^{7}$ She was first treated with hydroxyurea, then with ruxolitinib, which became available in our Institution starting from February 2013. In spite of an initial reduction of the spleen size and the remission of the systemic symptoms, on May 2014 ruxolitinib was withdrawn due to the reappearance of both painful splenomegaly and systemic symptoms, and the patient underwent splenectomy followed by treatment with hydroxyurea.

Although the Dynamic International Prognostic Scoring System (DIPSS) score was now intermediate- $2,{ }^{8}$ the HSCT was not performed because the only patient's brother was HLA haploidentical and no unrelated donors were unavailable. A few months later, a 10/12 matched male donor (antigen mismatch in DPB1) was identified and a peripheral blood hematopoietic stem cell (PBSC) HSCT could be scheduled. Patient and donor differed in $\mathrm{AB}$ blood group systems and CMV serostatus $(\mathrm{B}+v s$ 
A+, CMV IgG positive vs negative). The BM biopsy done before the HSCT was revealing that the patient's BM had a diffuse and intense fibrosis (Figure 1). ${ }^{9}$ At that time the DIPSS score was still intermediate-2, and the HSCT-comorbidity index was low. ${ }^{10}$ The HSCT was finally administered on March 2015 with a myeloablative conditioning consisting of busulfan $(16 \mathrm{mg} / \mathrm{kg}$ i.v.) and cyclophosphamide (120 mg/kg i.v.). The patient received $10.66 \times 10^{6} / \mathrm{kg}$ CD34+ cells, while the CD3+ cells infused were $24.47 \times 10^{6} / \mathrm{kg}$. Graft-versus-host disease (GVHD) prophylaxis included rabbit antithymocyte globulins, cyclosporine and short course methotrexate. In spite of the high number of CD34+ cells infused, on day +28 the patient was diagnosed with PGF. At that time an empirical antibacterial and antimycotic therapy was being delivered due to a persistent fever and the CTscan evidence of a single excavated lesion of 5-6 cm on the upper lobe of the left lung in the absence of galactomannan positivity.

The urgent requirement for a new donor and the rapid worsening of the patient's clinical condition (ECOG3) prompted us to schedule as a salvage treatment a PBSC HSCT from the haploidentical brother, who was $\mathrm{AB} 0$-mismatched $(\mathrm{A}+)$ and $\mathrm{CMV} \operatorname{IgG}$ positive. Therefore, on day +37 from the first HSCT, the patient received $6 \times 10^{6} / \mathrm{kg}$ $\mathrm{CD} 34+$ and $32.6 \times 10^{7} / \mathrm{kg} \mathrm{CD} 3+$ cells from the brother, following a conditioning regimen consisting of only fludarabine ( 30 $\mathrm{mg} / \mathrm{m}^{2}$ day for 3 days). On day +3 after the haploidentical HSCT the patient underwent cyclophosphamide (50 mg/kg i.v.) and starting from day +4 cyclosporine and mycophenolate mofetil as GVHD prophylaxis. G-CSF was given from day +5 and it was stopped on day +13 , when the engraftment was documented. Starting from the same time the patient presented a grade II skin GVHD that required steroid therapy until day +49 . Following the haploidentical HSCT, the BM aspirate was performed on day +21 , +90 , and +180 , showing a fulldonor chimerism by either fluorescence in situ hybridization with sex chromosomespecific DNA probes, and variable number tandem repeats analysis. Worthy of note, the BM biopsies performed on day +90 and +180 showed a progressive reduction of BM fibrosis as shown in Figure 1.

Importantly, the excavated lesion of the left lung was progressively decreasing and no GVHD was observed.

Unfortunately, on day +290 a relapse of disease was documented and the patient died of pulmonary infection few months later while in palliative treatment.

\section{Discussion}

The immediate availability of new donors for patients experiencing PGF is of critical importance and in this perspective the use of related haploidentical donors may offer obvious advantages. However, the new conditioning regimen together with the increased immunosuppression required to overcoming HLA disparity, may cause severe organ toxicities and infections. Recent reports have shown that haploidentical HSCT, either T-cell depleted, ${ }^{11,12}$ or unmanipulated, ${ }^{13-17}$ usually preceded by reduced intensity conditioning (RIC), can be safely and effectively administered to patients with PGF. However, the patients described in the aforementioned reports were mainly affected by acute or chronic myeloid leukemia, juvenile myelomonocytic leukemia, or myelodisplastic syndrome. ${ }^{11-17}$

To the best of our knowledge, the successful administration of unmanipulated haploidentical HSCTs has been previously described only in two MF patients with a follow-up of 30 and 11.5 months respectively, who had reported a PGF after an unrelated HLA full-matched RIC-HSCT. ${ }^{14}$ In both cases the 1-day conditioning regimen was composed of $30 \mathrm{mg} / \mathrm{m}^{2}$ fludarabine, $2 \mathrm{~g} / \mathrm{m}^{2}$ cyclophosphamide, $20 \mathrm{mg}$ alemtuzumab i.v., and 200 cGy total body irradiation, based on the assumption that the first preparative regimen might had already suppressed the recipient's immune systems. As for our patient, the conditioning scheme initially scheduled, ${ }^{18}$ actually included only fludarabine ( $30 \mathrm{mg} / \mathrm{m}^{2}$ for 3 days) given the poor patient's clinical status and the lack of any residual hematopoiesis at the BM biopsy of day +28 (not shown). Moreover, in contrast to the original schedule, ${ }^{18}$ we infused PBSCs instead of BM, due to the need of a rapid engraftment. Interestingly, in agreement with a recent study demonstrating a better outcome for PGF patients receiving $\mathrm{PBMCs}$ as compared to other stem cell sources, ${ }^{19}$ most of the PGF patients who underwent a haploidentical HSCT also received PBMCs. ${ }^{13-17}$

Worthy of note, in our patient the engraftment paralleled the progressive regression of BM fibrosis (Figure 1), therefore confirming previous experiences evidencing that a high fibrosis grading is not a major obstacle to HSCT in MF patients, but instead a dynamic process largely dependent on the microenvironment. ${ }^{6}$

As for our patient, the haploidentical HSCT was able to overcome the PGF and to reduce the $\mathrm{BM}$ fibrosis. However it turned out to be ineffective against the long-term control of the disease, thus suggesting that the conditioning regimen delivered and/or the transplant-related immune-mediated effect, were not capable of eradicating the disease.

\section{Conclusions}

Overall, given the limits of a single experience, this clinical case indicates that the unmanipulated haploidentical HSCT may be effective in rescuing MF patients from PGF. In addition, the higher number of PGF observed in patients undergoing unrelated HSCT, ${ }^{19}$ together with the difficulties of delivering a new conditioning regimen, support recent data indicating that the hap-

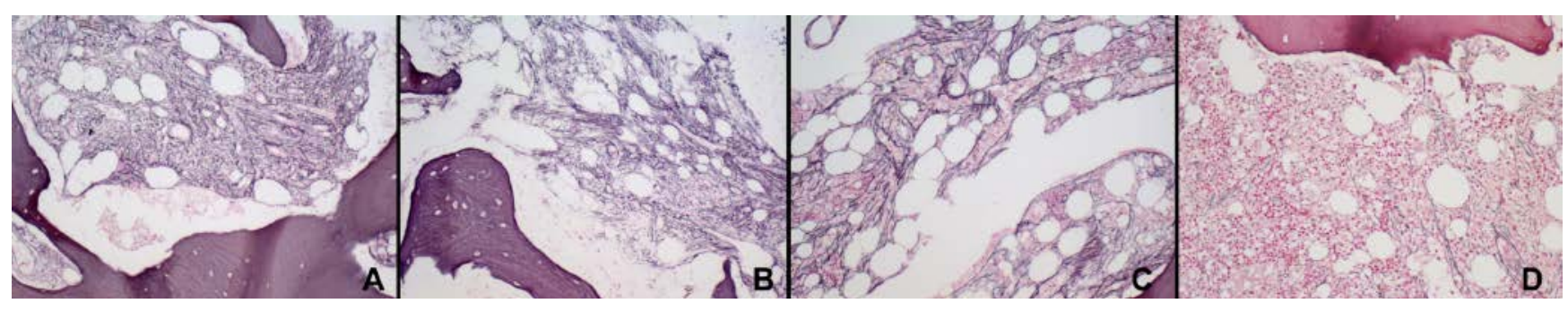

Figure 1. Grading of bone-marrow fibrosis (MF) according to the WHO guidelines using the Gomori stain. MF was evaluated: A) before the unrelated allogeneic HSCT, B) before the haploidentical HSCT, C) on day +90 and D) on day +180 following the haploidentical HSCT. Final magnification 200x. 
loidentical donor may be a reasonable first option for MF patients lacking a HLAmatched relative. ${ }^{20} \mathrm{We}$ are aware that only prospective studies aimed at comparing the outcome of recipients of conventional versus alternative donors will definitively establish the role of haploidentical HSCT in MF patients.

\section{References}

1. Cervantes F. How I treat myelofibrosis. Blood 2014;124:2635-42.

2. Tefferi A, Vannucchi AM. Genetic risk assessment in myeloproliferative neoplasms. Mayo Clin Proc 2017;92:128390.

3. Gupta V, Gotlib J, Radich JP, et al. Janus kinase inhibitors and allogeneic stem cell transplantation for myelofibrosis. Biol Blood Marrow Transplant 2014;20:1274-81.

4. Kroger NM, Deeg JH, Olavarria E, et al. Indication and management of allogeneic stem cell transplantation in primary myelofibrosis: a consensus process by an EBMT/ELN international working group. Leukemia 2015;29: 2126-33.

5. Olsson RF, Logan BR, Chaudhury S, et al. Primary graft failure after myeloablative allogeneic hematopoietic cell transplantation for hematologic malignancies. Leukemia 2015;29:1754-62.

6. Kroger N, Kvasnicka M, Thiele J. Replacement of hematopoietic system by allogeneic stem cell transplantation in myelofibrosis patients induces rapid regression of bone marrow fibrosis. Fibrogenesis Tissue Repair 2012;5:S25.

7. Cervantes F, Dupriez B, Pereira A, et al. New prognostic scoring system for primary myelofibrosis based on a study of the International Working Group for Myelofibrosis Research and Treatment. Blood 2009; 113:2895-901.

8. Passamonti F, Cervantes F, Vannucchi AM, et al. A dynamic prognostic model to predict survival in primary myelofibrosis: a study by the IWG-MRT (International Working Group for Myeloproliferative Neoplasms Research and Treatment). Blood 2010;115:17038.

9. Thiele J, Kvasnicka HM, Facchetti F, et al. European consensus on grading bone marrow fibrosis and assessment of cellularity. Haematologica 2005;90:112832.

10. Sorror ML, Maris MB, Storb R, et al. Hematopoietic cell transplantation (HCT)-specific comorbidity index: a new tool for risk assessment before allogeneic HCT. Blood 2005; 106:2912-9.

11. Moscardo F, Romero S, Sanz J, et al. T cell-depleted related HLA-mismatched peripheral blood stem cell transplantation as salvage therapy for graft failure after single unit unrelated donor umbilical cord blood transplantation. Biol Blood Marrow Transplant 2014;20:1060-3.

12. Park JA, Koh KN, Choi ES, et al. Successful rescue of early graft failure in pediatric patients using T-cell-depleted haploidentical hematopoietic SCT. Bone Marrow Transplant 2013;49:2705.

13. Yoshihara S, Ikegame K, Taniguchi K, et al. Salvage haploidentical transplantation for graft failure using reducedintensity conditioning. Bone Marrow Transplant 2011;47:369-73.

14. Kanda J, Horwitz ME, Long GD, et al. Outcomes of a 1-day non myeloablative salvage regimen for patients with primary graft failure after allogeneic hematopoietic cell transplantation. Bone Marrow Transplant 2012;47:700-5.

15. Tang BL, Zhu XY, Zheng CC, et al. Successful early unmanipulated haploidentical transplantation with reducedintensity conditioning for primary graft failure after cord blood transplantation in hematologic malignancy patients. Bone Marrow Transplant 2015;50:24852.

16. Perini GF, Kerbauy FR, Fernandes JF, et al. Haploidentical non-myeloablative stem cell transplantation as salvage for graft failure in a patient with juvenile myelomonocytic leukemia. Pediatr Blood Cancer 2011;57:1084.

17. Pagliuca S, Legendre H, Morin S, et al. Success of haploidentical hematopoietic stem cells transplantation in the treatment of graft failure. Ann Hematol 2016;95:353-4.

18. Luznik L, O’Donnell PV, Symons HJ, et al. HLA-haploidentical bone marrow transplantation for hematologic malignancies using non myeloablative conditioning and high-dose, post transplantation cyclophosphamide. Biol Blood Marrow Transplant 2008;14:641-50.

19. Fuji S, Nakamura F, Hatanaka K, et al. Peripheral blood as a preferable source of stem cells for salvage transplantation in patients with graft failure after cord blood transplantation: a retrospective analysis of the registry data of the Japanese Society for Hematopoietic Cell Transplantation. Biol Blood Marrow Transplant 2012;18:1407-14.

20. Bregante S, Dominietto A, Ghiso A, et al. Improved outcome of alternative donor transplantations in patients with myelofibrosis: from unrelated to haploidentical family donors. Biol Blood Marrow Transplant 2016;22:324-9. 